\title{
Ras-p53 genomic cooperativity as a model to investigate mechanisms of innate immune regulation in gastrointestinal cancers
}

\author{
Austin R. Dosch ${ }^{1,2}$, Walid K. Chatila ${ }^{3}$, Yuguang Ban ${ }^{4}$, Anna Bianchi ${ }^{1}$, Nilesh U. \\ Deshpande $^{1}$, Iago De Castro Silva ${ }^{1}$, Nipun B. Merchant ${ }^{1,2}$ and Jashodeep Datta ${ }^{1,2}$ \\ ${ }^{1}$ Division of Surgical Oncology, Dewitt Daughtry Department of Surgery, University of Miami Miller School of Medicine, Miami, \\ $\mathrm{FL}$, USA \\ ${ }^{2}$ Sylvester Comprehensive Cancer Center, Miami, FL, USA \\ ${ }^{3}$ Center for Molecular Oncology, Memorial Sloan Kettering Cancer Center, New York, NY, USA \\ ${ }^{4}$ Department of Public Health Sciences; University of Miami Miller School of Medicine, Miami, FL, USA \\ Correspondence to: Jashodeep Datta, email: jash.datta@med.miami.edu \\ Keywords: Ras-p53 cooperativity; Ras; TP53; immune; gastrointestinal cancer \\ Received: May 20, $2021 \quad$ Accepted: May 26, $2021 \quad$ Published: September 28, 2021
}

Copyright: $\odot 2021$ Dosch et al. This is an open access article distributed under the terms of the Creative Commons Attribution License (CC BY 3.0), which permits unrestricted use, distribution, and reproduction in any medium, provided the original author and source are credited.

\section{ABSTRACT}

Despite increasingly thorough mechanistic understanding of the dominant genetic drivers of gastrointestinal (GI) tumorigenesis (e.g., Ras/Raf, TP53, etc.), only a small proportion of these molecular alterations are therapeutically actionable. In an attempt to address this therapeutic impasse, our group has proposed an innovative extreme outlier model to identify novel cooperative molecular vulnerabilities in high-risk GI cancers which dictate prognosis, correlate with distinct patterns of metastasis, and define therapeutic sensitivity or resistance. Our model also proposes comprehensive investigation of their downstream transcriptomic, immunomic, metabolic, or upstream epigenomic cellular consequences to reveal novel therapeutic targets in previously "undruggable" tumors with high-risk genomic features. Leveraging this methodology, our and others' data reveal that the genomic cooperativity between Ras and p53 alterations is not only prognostically relevant in GI malignancy, but may also represent the incipient molecular events that initiate and sustain innate immunoregulatory signaling networks within the GI tumor microenvironment, driving T-cell exclusion and therapeutic resistance in these cancers. As such, deciphering the unique transcriptional programs encoded by Ras-p53 cooperativity that promote innate immune trafficking and chronic inflammatory tumor-stromal-immune crosstalk may uncover immunologic vulnerabilities that could be exploited to develop novel therapeutic strategies for these difficult-to-treat malignancies.

\section{INTRODUCTION}

The widespread availability and frequent inclusion of high throughput genomic sequencing technology in cancer medicine has enabled comprehensive characterization of the pan-cancer mutational landscape. Moreover, knowledge of the molecular underpinnings of tumorigenesis has accelerated drug discovery targeting dominant oncogenic drivers in specific cancers-ushering in the era of "precision oncology" - as well discovery of novel biomarkers that predict such drug responses and/ or inform prognosis. These valuable insights into the "genotype-phenotype chasm"-i.e., how these molecular alterations dictate tumor biology - are particularly relevant in gastrointestinal (GI) malignancies, where outcomes with standard multimodality cancer treatment (e.g., surgery, chemotherapy, radiotherapy, etc.) continue to be dismal compared with many other solid cancers [1]. However, despite thorough mechanistic understanding of the dominant genetic drivers of GI tumorigenesis, 
including oncogenic Ras/Raf activation, deleterious TP53 mutations resulting in p53 inactivation, loss of SMAD4, etc., only a small proportion of these molecular alterations are therapeutically actionable [2].

In an attempt to address this therapeutic impasse, our group has proposed an innovative model to decipher novel molecular vulnerabilities in high-risk GI cancers using an extreme outlier approach, highlighted in several recently published manuscripts. In this model, we aim to identify co-occurrent alterations between critical oncogenic drivers that are not only associated with extremes of oncologic outcome (i.e., survival, pathologic response, etc.) but also dictate prognosis [3, 4], correlate with distinct patterns of metastasis [3], and define therapeutic sensitivity or resistance [5]. Beyond simply identifying these cooperative molecular alterations that underlie highrisk clinical phenotypes in GI cancers, our model also proposes comprehensive investigation of their downstream transcriptomic, immunomic, metabolic, or upstream epigenomic cellular consequences [6]. We hypothesize that the iterative investigative journey from extreme outlier clinical phenotypes to identification of underlying highrisk genotypes to discovery of multi-omic repercussions of these alterations in GI malignancies will reveal novel therapeutic targets in previously "undruggable" tumors with high-risk genomic features.

Leveraging this methodology, our group is actively pursuing the genomic cooperativity between Ras and p53 alterations in GI malignancy. In a recent manuscript in "Clinical Cancer Research", we utilized an extremes-ofsurvivorship approach in patients with resected colorectal cancer liver metastasis (CRLM) and demonstrated that concurrent mutations in both Ras pathway and TP53 alterations were significantly more frequent in $\leq 2$-year survivors, whereas co-altered Ras-TP53 was absent in $\geq 10$-year survivors $(67 \%$ vs. $0 \%, P<0.001)$ [3]. In a separate manuscript recently published in "Annals of Surgery", Ras-p53 cooperative mutations defined systemic and liver-directed chemotherapy resistance in patients with unresectable CRLM and was independently associated with worse survival (HR 2.52, 95\% CI:1.374.64, $p=0.003$ ) after controlling for conversion to surgical resection, liver metastasis burden, preoperative extrahepatic disease, and use of chemotherapy [5]. In yet another manuscript, currently in press [Narayan R, Datta $\mathrm{J}$ et al., personal communication], Ras-TP53 cooperativity was associated with earlier local and distant recurrence in CRLM patients undergoing complete resection followed by adjuvant systemic and liver-directed chemotherapy. Not only is Ras-p53 genomic cooperativity oncologically significant, but also has broad clinical relevance by virtue of its frequent occurrence in colorectal cancers, with studies reporting concurrent Ras-p53 mutations occurring in nearly one-third of patients with colorectal liver metastasis [7]. Published data from the MSKCC database (publicly available through http://www.cbioportal.org) corroborate these statistics [8], with Ras-p53 cooperative alterations present in nearly a third of sequenced patients (Figure 1A). Collectively, these observations not only provide novel insight into the biologic relevance of Ras-p53 cooperativity in the clinical arena, but also establish Ras-p53 cooperativity as a high-risk genomic subgroup of GI cancers that manifest a clinical phenotype defined by chemoresistance, aggressive non-salvageable metastatic proclivity, and dismal cancer-related survival.

Data from The Cancer Genome Atlas (TCGA) also demonstrate that the RTK-Ras and p53 oncogenic pathways are co-altered in a substantial portion of patients with non-colorectal gastrointestinal cancers, particularly pancreatic ductal adenocarcinoma (PDAC) in which more than half of all patients demonstrate Ras-p53 cooperative alterations (Figure 1B). These data are particularly relevant because Ras-p53 cooperativity represents a foundational molecular event in GI tumorigenesis, modulating cellular signaling to induce the spontaneous development of invasive cancers in well-established genetically-engineered mouse models (GEMM) [911]. In-depth analysis in these sophisticated models suggest an interdependence on activating Ras pathway mutations and p53 loss in order to bypass cell-intrinsic mechanisms that abrogate tumor growth [12], and perhaps explain why mutations in these two specific pathways occur simultaneously in GI cancer patients. In addition, inactivating mutations in p53 not only abolish its ability to bind consensus DNA sequences and transactivate p53 target genes, but also enable the mutant p53 protein to acquire new oncogenic properties that are independent of wild-type p53 [13], further engendering Ras-p53 oncogenic cooperation. Beyond sufficiency to induce spontaneous tumorigenesis, Ras-p53 cooperativity also promotes invasion and motility and generates a highly metastatic phenotype in vivo [14, 15]. Recent insight into the molecular underpinnings of the pro-metastatic phenotype generated by Ras-p53 cooperativity revealed that oncogenic KRAS effectors activate CREB1 to allow physical interactions with mutant p53 that hyperactivate multiple pro-metastatic transcriptional networks. Moreover, mutant p53 and CREB1 upregulate the prometastatic transcription factor $F O X A 1$, while promoting Wnt $/ \beta$-catenin signaling, together driving metastasis [16]. Finally, oncogenic cooperativity between Ras and p53 signaling is also mechanistically supported by the phenomenon of oncogene-induced senescence, whereby activating Ras pathway mutations induce a senescent program that results in replicative arrest of tumor cells [17]. Interestingly, this Ras-induced senescent program can be overcome by p53 inactivation (or p16 loss), leading to continued proliferation and escape from senescenceinduced growth inhibition [18, 19]. Therefore, multiple lines of evidence underscore the critical role of Ras-p53 cooperativity in driving aggressive clinical phenotypes in GI cancer. 
While the prognostic relevance of Ras-p53 genomic cooperativity and its mechanistic underpinnings are undoubtedly compelling, neither mutant Ras nor p53 are yet considered therapeutically actionable. As mentioned previously, we believe that deciphering how these cooperative driver mutations orchestrate tumorpromoting and immunosuppressive tumor-stromalimmune interactions in the tumor microenvironment (TME) to promote treatment resistance may reveal novel therapeutic opportunities for these aggressive cancers. As such, our group is particularly interested in understanding and targeting novel immune repercussions of Ras-p53 cooperativity in GI cancers. While the independent roles of both oncogenic Ras activation and p53 loss in establishing pro-inflammatory signaling and the recruitment and activation of immunosuppressive cells is well established [20-22], how Ras-p53 cooperativity encode unique tumor cell-intrinsic transcriptional programs to promote immunologic remodeling of the TME in gastrointestinal cancers is incompletely understood. Early insight into such coordinated tumor-intrinsic programs driven by Ras-p53 cooperativity came from an elegant study by McMurray and colleagues in which murine colon cells containing individual or combined mutants of p53 $\left(\mathrm{p} 53^{\mathrm{R} 175 \mathrm{H}}\right)$ or activated H-Ras $\left(\mathrm{Ras}^{\mathrm{G} 12 \mathrm{~V}}\right)$ were profiled by microarray analysis to delineate a set of gene transcripts - encompassing a broad range of functional annotations such as signal transduction, metabolism, cell adhesion, etc. - that are synergistically regulated by Ras-p53 cooperative signaling [23]. Further investigation demonstrated that a majority of these genes are critical for tumor development, as evident by attenuation of tumor growth in mice following gene perturbation experiments.

In a complementary study, Buganim and colleagues utilized an in vitro transformation model using immortalized human fibroblasts transduced with $\mathrm{Ras}^{\mathrm{G} 12 \mathrm{~V}}$ and $\mathrm{p} 53^{\mathrm{R} 175 \mathrm{H}}$ constructs to identify an inflammationassociated gene signature synergistically induced by cooperative Ras-p53 alterations [24]. Among these were several genes related to innate immune cell recruitment and activation such as ELR chemokines (i.e., $C X C L 1, C X C L 2$, $C X C L 3$, and $C X C L 6)$ and well as the pro-inflammatory ligands (i.e., IL1B, IL6, IL8, and CSF2) (Table 1). To explore these intriguing data further, we performed an analysis of whole tumor transcriptomic data from the colon (COAD) and rectal cancer (READ) datasets of the TCGA. Using established single-cell immune deconvolution pipelines (ImmuneCellAI [25]), we compared the computationally inferred immune populations in primary COAD and READ tumors harboring concurrent hotspot alterations in Ras (i.e., $K R A S, N R A S$, or $B R A F$ ) and p53 versus tumors with Ras alterations alone. Ras-p53 cooperative COAD/READ tumors exhibited increases in immunosuppressive innate immune populations

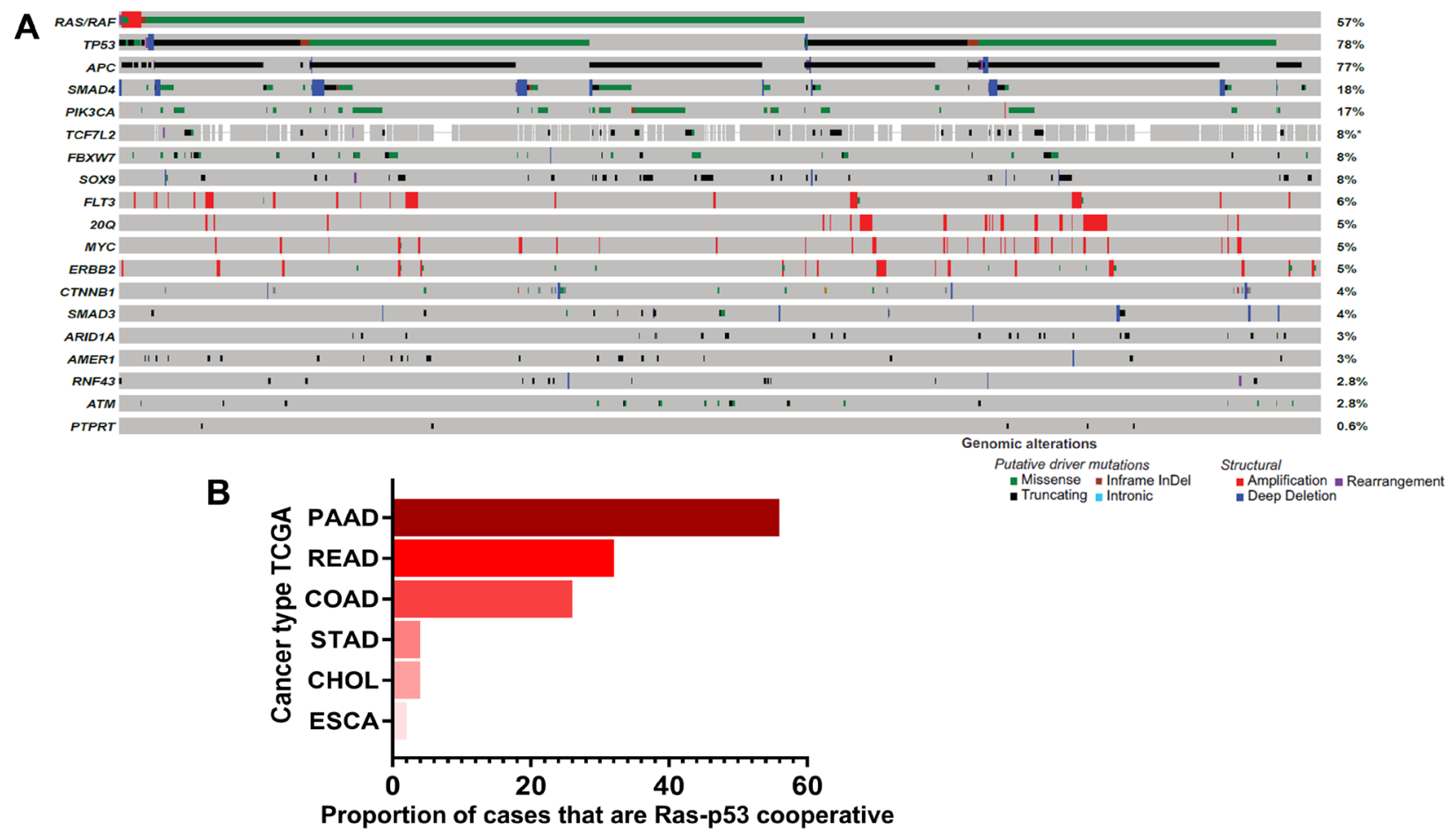

Figure 1: Ras-p53 genomic cooperativity in gastrointestinal cancer. (A) Oncoprint demonstrating most frequent putative driver alterations in patients with colorectal cancer in the MSK-IMPACT Clinical Sequencing Cohort (available through http://www.cbioportal. org). Gene names are provided to the left and mutational frequencies to the right. Genomic alterations are classified as putative driver or structural alterations in adjoining legend. (B) Frequency of co-altered Ras pathway and p53 alterations were determined in patients with pancreatic cancer (PAAD), rectal cancer (READ), colon cancer (COAD), stomach cancer (STAD), cholangiocarcinoma (CHOL), and esophageal (ESCA) cancer in The Cancer Genome Atlas (TCGA) dataset. 
Table 1: Differentially overexpressed transcripts associated with innate immunity induced by cooperative Ras-p53 mutations

\section{ELR Chemokines}

CXCL1

CXCL2

CXCL3

CXCL6

\begin{tabular}{l}
\hline Pro-Inflammatory Ligands \\
\hline IL1B \\
IL6 \\
IL8 \\
CSF2
\end{tabular}

Extracellular Matrix-related Genes

$M M P 3$

CLECSF2

Data from Buganim et al. demonstrating increased expression of selected chemokines (i.e., CXCL1, CXCL2, CXCL3, and $C X C L 6$ ) and pro-inflammatory ligands (i.e., IL1B, IL6, IL8, and CSF2) from immortalized human fibroblasts transduced with $\mathrm{Ras}^{\mathrm{G} 12 \mathrm{~V}}$ and $\mathrm{p} 53^{\mathrm{R} 175 \mathrm{H}}$ mutant constructs [24].

such as tumor-associated neutrophils, tumor-associated macrophages (TAMs), monocytes, inducible T-regulatory type $1(\operatorname{Tr} 1)$ cells, and $\gamma \delta$ T-cells (Figure $2 \mathrm{~A}$ and $2 \mathrm{~B}$ ). Recent findings from a study by Blagih and colleagues expand on these hypothesis-generating data in in vivo models of GI cancer to indicate an association between cooperative Ras-p53 alterations and the recruitment of innate immune populations into established tumors. In this study, concurrent KRAS and TP53 mutations coordinated the influx of myeloid cells into tumors, but did not affect adaptive immune subsets such as regulatory T-cells. Using an inducible $K R A S^{G / 2 D /+}$ construct in PDAC tumor cells
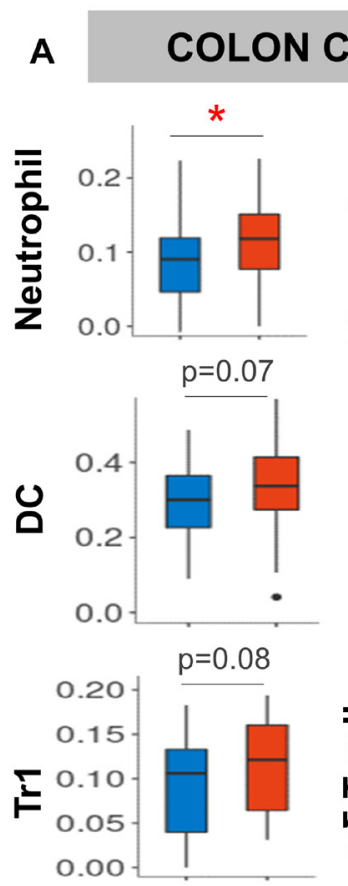

\section{ANCER}
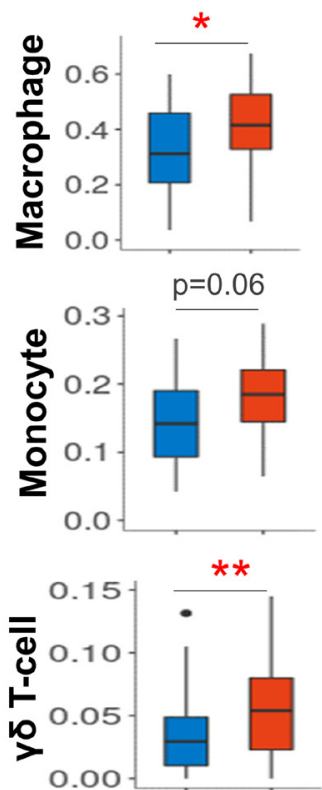
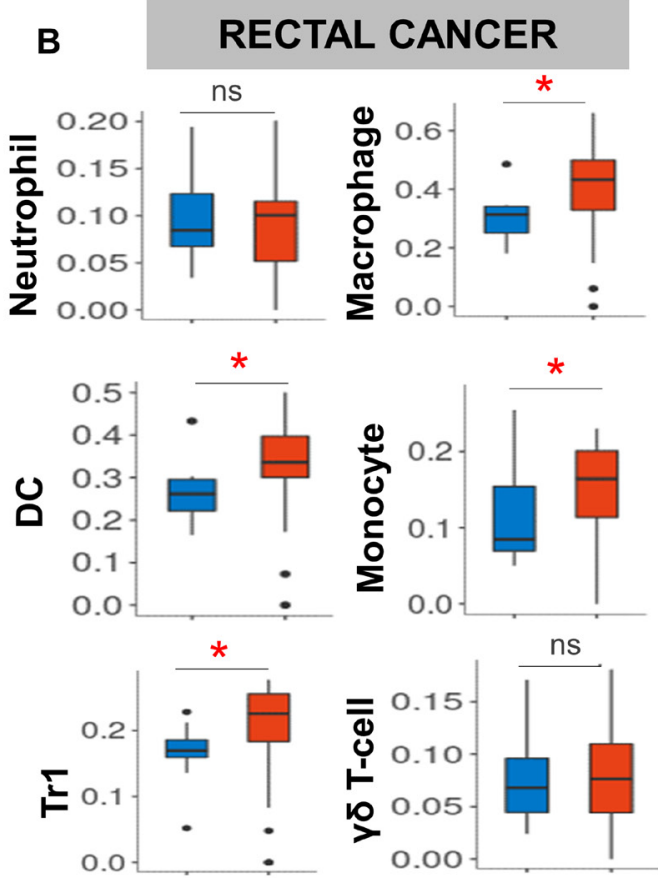

Figure 2: Ras-p53 cooperative mutations in rectal and colon cancer are associated with an innate immunoregulatory phenotype. Single-cell immune deconvolution from bulk RNA sequencing data from colon and rectal cancer cases curated from The Cancer Genome Atlas (TCGA) Pan-Cancer database was performed using ImmuneCellAI as described in Materials and Methods. (A and B) Comparison of neutrophil, macrophage, dendritic cell (DC), monocyte, inducible T regulatory type 1 ( $\operatorname{Tr} 1$ ), and $\gamma \delta$ T-cell signatures between Ras-alone (blue) and Ras-p53 co-operative (red) (A) colon and (B) rectal cancer samples in the TCGA database. ns, not significant; ${ }^{*} p<0.05 ;{ }^{* *} p<0.01$. 
containing biallelic loss of TP53, the authors demonstrated that withdrawal of $K R A S$ mutations resulted in a significant reduction in the levels of intratumoral $\mathrm{CD} 11 \mathrm{~b}^{+}$myeloid cells, CD11b ${ }^{+} \mathrm{F} 4 / 80^{+}$macrophages, and $\mathrm{CD} 11 \mathrm{~b}^{+} \mathrm{CXCR}^{+}$ tumor-associated myeloid cells, highlighting the importance of cooperative Ras-p53 mutations in driving innate immune cell recruitment in pancreatic cancer [26]. These findings strongly link concurrent Ras-p53 mutations with the establishment of innate immunoregulatory signaling within GI tumors and may represent the molecular events initiating the recruitment and activation of innate immune cells that sustain immunosuppression within the GI cancer TME.

Taken together, prior and emerging data indicate that Ras-p53 genomic cooperativity is an ideal model to investigate mechanisms of innate immune regulation in gastrointestinal cancers. Innate immune populations such as neutrophils, neutrophilic myeloid-derived suppressor cells (MDSCs), and TAMs are not only critically important for the initiation and progression of solid organ cancers [27], but have also been extensively implicated in dictating responses to chemotherapy and/or immunotherapy in GI cancers, making efforts to further understand and characterize the mechanisms governing their influx into and function within the TME critical to improving patient outcomes [28-31]. As such, while is appears clear that Ras-p53 cooperativity is associated with innate immune trafficking into the GI TME, the specific transcriptional programs encoded by Ras-p53 cooperativity that govern innate immunoregulation in the TME have not been extensively explored. Ongoing efforts by our group are focused on comprehensively understanding the diverse transcriptional programs encoded by cooperative Ras-p53 alterations in GI cancers that promote chronic inflammatory tumor-stromal-immune crosstalk, innate immune trafficking, immune exclusion, and therapeutic resistance. We believe that these investigations will uncover novel immunologic vulnerabilities that could be exploited to develop therapeutic strategies to mitigate these immunomodulatory effects, overcome immune exclusion and therapeutic resistance, unleash anti-tumor immunity, and ultimately revolutionize treatment for patients with these difficult-to-treat GI cancers.

\section{MATERIALS AND METHODS}

Previously published genomic data [3, 8] was curated from http://www.cbioportal.org [32, 33] and depicted using an oncoprint to visualize the frequency of driver, putative driver, and variants of undetermined significance mutations in all patients with colorectal cancer $(n=935)$ at MSKCC that had undergone sequencing using the MSK-IMPACT platform.

The TCGA pan-cancer database was queried for all hotspot mutations in Ras family members (KRAS, NRAS, $H R A S$ ) and TP53, and the frequency of co-altered Ras-
TP53 mutations across selected GI cancers was tabulated. TCGA samples in the COAD and READ datasets were dichotomized into Ras-p53 cooperative and Ras-alone altered, and resulting fragments per kilobase of transcript per million (FPKM) reads from these two groups were entered into a publicly available single cell immune deconvolution pipeline ImmuneCellAI (available at: http://bioinfo.life.hust.edu.cn/ImmuCellAI\#!/) [25]. ImmuneCellAI estimates the abundance of 24 immune cells from gene expression datasets, comprising $18 \mathrm{~T}$-cell subtypes and 6 other immune cells: B-cell, NK cells, Monocyte, Macrophage, Neutrophil, and DC. Results from selected immune sub-populations in both COAD and READ datasets were depicted as box-and-whiskers plots.

\section{CONFLICTS OF INTEREST}

The authors declare no conflicts of interest.

\section{FUNDING}

The authors acknowledge support from the National Cancer Institute (NCI) of the National Institutes of Health (NIH) Award P30 CA240139 (to J. Datta and N.B. Merchant); KL2 career development grant by the Miami Clinical and Translational Science Institute (CTSI) under NIH Award UL1TR002736, American College of Surgeons Franklin Martin Career Development Award, and Association for Academic Surgery Joel J. Roslyn Faculty Award (to J. Datta); AR. Dosch is supported by NCI/NIH Award T32CA211034 (to N.B. Merchant).

\section{REFERENCES}

1. Siegel RL, Miller KD, Jemal A. Cancer statistics, 2020. CA Cancer J Clin. 2020; 70:7-30. https://doi.org/10.3322/ caac. 21590. [PubMed]

2. Iacobuzio-Donahue CA. Genetic evolution of pancreatic cancer: lessons learnt from the pancreatic cancer genome sequencing project. Gut. 2012; 61:1085-94. https://doi. org/10.1136/gut.2010.236026. [PubMed]

3. Datta J, Smith JJ, Chatila WK, McAuliffe JC, Kandoth C, Vakiani E, Frankel TL, Ganesh K, Wasserman I, LipsycSharf M, Guillem J, Nash GM, Paty PB, et al. Coaltered Ras/B-raf and TP53 Is Associated with Extremes of Survivorship and Distinct Patterns of Metastasis in Patients with Metastatic Colorectal Cancer. Clin Cancer Res. 2020; 26:1077-85. https://doi.org/10.1158/1078-0432.CCR-192390. [PubMed]

4. Datta J, Da Silva EM, Kandoth C, Song T, Russo AE, Hernandez JM, Taylor BS, Janjigian YY, Tang LH, Solit DB, Strong VE. Poor survival after resection of early gastric cancer: extremes of survivorship analysis reveal distinct genomic profile. Br J Surg. 2020; 107:14-19. https://doi. org/10.1002/bjs.11443. [PubMed] 
5. Datta J, Narayan RR, Goldman DA, Chatila WK, Gonen M, Strong J, Balachandran VP, Drebin JA, Kingham TP, Jarnagin WR, Schultz N, Kemeny NE, DAngelica MI. Distinct Genomic Profiles are Associated With Conversion to Resection and Survival in Patients With Initially Unresectable Colorectal Liver Metastases Treated With Systemic and Hepatic Artery Chemotherapy. Ann Surg. 2020. https://doi.org/10.1097/SLA.0000000000004613. [PubMed]

6. Dosch AR, Mehra S, Merchant NB, Datta J. Deciphering high risk molecular alterations in gastrointestinal malignancy utilizing an extreme outlier strategy. Oncoscience. 2020; 7:26-29. https://doi.org/10.18632/ oncoscience.503. [PubMed]

7. Chun YS, Passot G, Yamashita S, Nusrat M, Katsonis P, Loree JM, Conrad C, Tzeng CD, Xiao L, Aloia TA, Eng C, Kopetz SE, Lichtarge O, Vauthey JN. Deleterious Effect of RAS and Evolutionary High-risk TP53 Double Mutation in Colorectal Liver Metastases. Ann Surg. 2019; 269:917-23. https://doi.org/10.1097/SLA.0000000000002450. [PubMed]

8. Yaeger R, Chatila WK, Lipsyc MD, Hechtman JF, Cercek A, Sanchez-Vega F, Jayakumaran G, Middha S, Zehir A, Donoghue MTA, You D, Viale A, Kemeny N, et al. Clinical Sequencing Defines the Genomic Landscape of Metastatic Colorectal Cancer. Cancer Cell. 2018; 33:125-36.e3. https://doi.org/10.1016/j.ccell.2017.12.004. [PubMed]

9. Hingorani SR, Wang L, Multani AS, Combs C, Deramaudt TB, Hruban RH, Rustgi AK, Chang S, Tuveson DA. Trp53R172H and KrasG12D cooperate to promote chromosomal instability and widely metastatic pancreatic ductal adenocarcinoma in mice. Cancer Cell. 2005; 7:46983. https://doi.org/10.1016/j.ccr.2005.04.023. [PubMed]

10. Westphalen CB, Olive KP. Genetically engineered mouse models of pancreatic cancer. Cancer J. 2012; 18:502-10. https://doi.org/10.1097/PPO.0b013e31827ab4c4. [PubMed]

11. Hill MA, Alexander WB, Guo B, Kato Y, Patra K, ODell MR, McCall MN, Whitney-Miller CL, Bardeesy N, Hezel AF. Kras and Tp53 Mutations Cause Cholangiocyte- and Hepatocyte-Derived Cholangiocarcinoma. Cancer Res. 2018; 78:4445-51. https://doi.org/10.1158/0008-5472. CAN-17-1123. [PubMed]

12. Courtois-Cox S, Jones SL, Cichowski K. Many roads lead to oncogene-induced senescence. Oncogene. 2008; 27:280109. https://doi.org/10.1038/sj.onc.1210950. [PubMed]

13. Buganim Y, Rotter V. p53: balancing tumour suppression and implications for the clinic. Eur J Cancer. 2009; 45:217-34. https://doi.org/10.1016/S0959-8049(09)70037-1. [PubMed]

14. Zheng S, El-Naggar AK, Kim ES, Kurie JM, Lozano G. A genetic mouse model for metastatic lung cancer with gender differences in survival. Oncogene. 2007; 26:6896-904. https://doi.org/10.1038/sj.onc.1210493. [PubMed]

15. Solomon H, Brosh R, Buganim Y, Rotter V. Inactivation of the p53 tumor suppressor gene and activation of the Ras oncogene: cooperative events in tumorigenesis. Discov Med. 2010; 9:448-54. [PubMed]
16. Kim MP, Li X, Deng J, Zhang Y, Dai B, Allton KL, Hughes TG, Siangco C, Augustine JJ, Kang Y, McDaniel JM, Xiong S, Koay EJ, et al. Oncogenic KRAS recruits an expansive transcriptional network through mutant p53 to drive pancreatic cancer metastasis. Cancer Discov. 2021; candisc.1228.2020. https://doi.org/10.1158/2159-8290.CD20-1228. [PubMed]

17. Braig M, Schmitt CA. Oncogene-induced senescence: putting the brakes on tumor development. Cancer Res. 2006; 66:2881-84. https://doi.org/10.1158/0008-5472. CAN-05-4006. [ [PubMed]

18. Morton JP, Timpson P, Karim SA, Ridgway RA, Athineos D, Doyle B, Jamieson NB, Oien KA, Lowy AM, Brunton VG, Frame MC, Evans TR, Sansom OJ. Mutant p53 drives metastasis and overcomes growth arrest/senescence in pancreatic cancer. Proc Natl Acad Sci U S A. 2010; 107:246-51. https://doi.org/10.1073/pnas.0908428107. [PubMed]

19. Serrano M, Lin AW, McCurrach ME, Beach D, Lowe $\mathrm{SW}$. Oncogenic ras provokes premature cell senescence associated with accumulation of p53 and p16INK4a. Cell. 1997; 88:593-602. https://doi.org/10.1016/s0092$\underline{\text { 8674(00)81902-9. }}$ [ [PubMed]

20. Hamarsheh S, Gross O, Brummer T, Zeiser R. Immune modulatory effects of oncogenic KRAS in cancer. Nat Commun. 2020; 11:5439. https://doi.org/10.1038/s41467020-19288-6. [PubMed]

21. Cui Y, Guo G. Immunomodulatory Function of the Tumor Suppressor p53 in Host Immune Response and the Tumor Microenvironment. Int J Mol Sci. 2016; 17:1942. https:// doi.org/10.3390/ijms17111942. [PubMed]

22. Ancrile BB, O'Hayer KM, Counter CM. Oncogenic rasinduced expression of cytokines: a new target of anticancer therapeutics. Mol Interv. 2008; 8:22-27. https://doi. org $/ 10.1124 / \mathrm{mi} .8 \cdot 1.6$. [PubMed]

23. McMurray HR, Sampson ER, Compitello G, Kinsey C, Newman L, Smith B, Chen SR, Klebanov L, Salzman P, Yakovlev A, Land H. Synergistic response to oncogenic mutations defines gene class critical to cancer phenotype. Nature. 2008; 453:1112-16. https://doi.org/10.1038/ nature06973. [PubMed]

24. Buganim Y, Solomon H, Rais Y, Kistner D, Nachmany I, Brait M, Madar S, Goldstein I, Kalo E, Adam N, Gordin M, Rivlin N, Kogan I, et al. p53 Regulates the Ras circuit to inhibit the expression of a cancer-related gene signature by various molecular pathways. Cancer Res. 2010; 70:2274-84. https://doi.org/10.1158/0008-5472.CAN-092661. [PubMed]

25. Miao YR, Zhang Q, Lei Q, Luo M, Xie GY, Wang H, Guo AY. ImmuCellAI: A Unique Method for Comprehensive T-Cell Subsets Abundance Prediction and its Application in Cancer Immunotherapy. Adv Sci (Weinh). 2020; 7:1902880. https://doi.org/10.1002/advs.201902880. [PubMed]

26. Blagih J, Zani F, Chakravarty P, Hennequart M, Pilley S, Hobor S, Hock AK, Walton JB, Morton JP, Gronroos 
E, Mason S, Yang M, McNeish I, et al. Cancer-Specific Loss of p53 Leads to a Modulation of Myeloid and T Cell Responses. Cell Rep. 2020; 30:481-96.e6. https://doi. org/10.1016/i.celrep.2019.12.028. [PubMed]

27. Hagerling C, Casbon AJ, Werb Z. Balancing the innate immune system in tumor development. Trends Cell Biol. 2015; 25:214-20. https://doi.org/10.1016/j.tcb.2014.11.001. [PubMed]

28. Sharma P, Hu-Lieskovan S, Wargo JA, Ribas A. Primary, Adaptive, and Acquired Resistance to Cancer Immunotherapy. Cell. 2017; 168:707-23. https://doi. org/10.1016/j.cell.2017.01.017. [PubMed]

29. Gabrilovich DI. Myeloid-Derived Suppressor Cells. Cancer Immunol Res. 2017; 5:3-8. https://doi.org/10.1158/23266066.CIR-16-0297. [PubMed]

30. Law AMK, Valdes-Mora F, Gallego-Ortega D. MyeloidDerived Suppressor Cells as a Therapeutic Target for Cancer. Cells. 2020; 9:561. https://doi.org/10.3390/cells9030561. [PubMed]
31. Pathria P, Louis TL, Varner JA. Targeting Tumor-Associated Macrophages in Cancer. Trends Immunol. 2019; 40:310-27. https://doi.org/10.1016/j.it.2019.02.003. [PubMed]

32. Cerami E, Gao J, Dogrusoz U, Gross BE, Sumer SO, Aksoy BA, Jacobsen A, Byrne CJ, Heuer ML, Larsson E, Antipin Y, Reva B, Goldberg AP, et al. The cBio cancer genomics portal: an open platform for exploring multidimensional cancer genomics data. Cancer Discov. 2012; 2:401-04. https://doi.org/10.1158/2159-8290.CD-12-0095. [PubMed]

33. Gao J, Aksoy BA, Dogrusoz U, Dresdner G, Gross B, Sumer SO, Sun Y, Jacobsen A, Sinha R, Larsson E, Cerami E, Sander C, Schultz N. Integrative analysis of complex cancer genomics and clinical profiles using the cBioPortal. Sci Signal. 2013; 6:pl1. https://doi.org/10.1126/ scisignal.2004088. [PubMed] 